\title{
Coumarin luciferins and mutant luciferases for robust multicompo- nent bioluminescence imaging
}

\author{
Zi Yao ${ }^{1, \ddagger}$, Donald R. Caldwell ${ }^{4, \$}$, Anna C. Love ${ }^{1, \$}$, Bethany Kolbaba-Kartchner ${ }^{5,6}$, Jeremy H. Mills ${ }^{5,6}$, \\ Martin J. Schnermann ${ }^{4,}$, Jennifer A. Prescher ${ }^{1,2,3, *}$ \\ ${ }^{1}$ Department of chemistry, ${ }^{2}$ Molecular Biology \& Biochemistry, and ${ }^{3}$ Pharmaceutical Sciences, University of California, Ir- \\ vine, Irvine CA, United States \\ ${ }^{4}$ Chemical Biology Laboratory, Center for Cancer Research, National Cancer Institute, Frederick MD, United States \\ ${ }^{5}$ School of Molecular Sciences, and ${ }^{6}$ The Biodesign Center for Molecular Design and Biomimetics, Arizona State University, \\ Tempe AZ, United States \\ *Authors to whom correspondence should be addressed.
}

$\ddagger$ These authors contributed equally to the work.

\begin{abstract}
Multicomponent bioluminescence imaging requires an expanded collection luciferase-luciferin pairs that emit farred or near-infrared light. Toward this end, we prepared a new class of luciferins based on a red-shifted coumarin scaffold. These probes (CouLuc-1s) were accessed in a two-step sequence via direct modification of commercial dyes. The bioluminescent properties of the CouLuc-1 analogs were also characterized, and complementary luciferase enzymes were identified using a two-pronged screening strategy. The optimized enzyme-substrate pairs displayed robust photon outputs and emitted a significant portion of nearinfrared light. The CouLuc-1 scaffolds are also structurally distinct from existing probes, enabling rapid multicomponent imaging. Collectively, this work provides novel bioluminescent tools along with a blueprint for crafting additional probes for multiplexed imaging.
\end{abstract}

\section{INTRODUCTION}

Bioluminescent enzymes (luciferases) are among the most popular reporters for imaging biological processes in vitro, in live cells, and in animal models. ${ }^{1-2}$ Luciferases generate light via the oxidation of small molecule luciferins (Figure 1a). Since no external light source is needed, bioluminescent probes offer high signal-to-noise ratios in heterogeneous environments. $^{3-5}$ The remarkable sensitivity, combined with the broad dynamic range, has made bioluminescence a go-to imaging technique for tracking cell movements, proliferation, and numerous other features in living organisms. ${ }^{6-8}$

While powerful, bioluminescence has been slow to transition to imaging multiple targets simultaneously, owing to a lack of distinguishable probes. ${ }^{9}$ Multiplexed imaging is possible using spectrally resolved luciferase-luciferin pairs. ${ }^{10-13}$ Indeed, several luciferin analogs have been developed that emit different colors of light. ${ }^{14-16}$ The emission profiles can be further tuned with engineered luciferases ${ }^{17-18}$ or luciferasefluorescent probe fusions. ${ }^{19-22}$ In many cases, though, the spectral resolution achieved is insufficient for routine application in vivo. Sensitive imaging in whole organisms requires $>650$ nm light, as these wavelengths are significantly less absorbed by tissue. ${ }^{23}$ However, few bioluminescent probes emit light in the requisite far-red to near-infrared (NIR) range. The perceived color of emission in traditional bioluminescent detec- tion also changes with the depth of the source, complicating the assignment of different colored probes. ${ }^{24}$

a

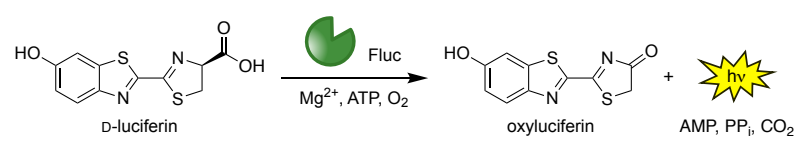

b

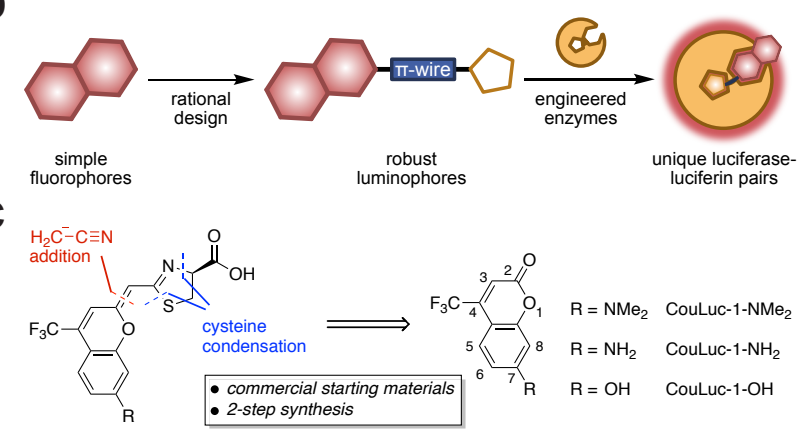

Figure 1. Red-emitting orthogonal bioluminescent probes designed from fluorophores. (a) D-Luciferin is oxidized by firefly luciferase (Fluc) to produce oxyluciferin and a photon of light. (b) Coumarin fluorophores were used as templates for red-shifted luciferins. (c) Retrosynthetic analysis of the CouLuc-1 analogs. 
Multiplexed bioluminescence imaging can also be achieved via substrate resolution - using luciferases that recognize different luciferin structures (i.e., orthogonal pairs). ${ }^{25}$ Light is produced when complementary enzymes and substrates react, but minimized in all other cases. ${ }^{26-28} \mathrm{~A}$ handful of such orthogonal probes have been co-opted for dual imaging in vivo, but applications have been mostly limited to monitoring superficial targets. ${ }^{29-31}$ This is due, in part, to a shortage of luciferins with both sufficient red emission and distinct molecular architectures. Candidate luciferins must also be sufficiently bright, bioavailable, and easy to synthesize — criteria that few existing probes meet. ${ }^{32-33}$ Consequently, routine multicomponent imaging with three or more red-emitting bioluminescent reporters remains challenging.

We surmised that unique classes of NIR-emitting luciferins could be developed using fluorescent scaffolds as guides (Figure $1 \mathrm{~b}$ ). Our approach was inspired by previous reports of luciferin analogs comprising entirely new heterocycles, ${ }^{34-35}$ including benzothiophene ${ }^{36}$, quinoline ${ }^{37}$, and coumarin derivatives. ${ }^{38}$ We also took cues from recent efforts to develop redshifted luciferins by extending the $\pi$-conjugation ${ }^{39-41}$ of the scaffold and restricting conformational flexibility (e.g., AkaLumine and infraluciferin). ${ }^{42-44}$ In most cases, the engineered luciferins were found to be poor substrates for Fluc. Light emission could be recouped, though, via extensive enzyme engineering (e.g., generating Akaluc and CBR mutants). ${ }^{39,44-45}$

We focused on a new class of luciferins (CouLuc-1s) comprising both an elongated $\pi$-system and a 4trifluoromethylcoumarin unit (Figure 1c). The coumarin fluorophore is a well-established imaging agent ${ }^{46-47}$ and has been incorporated into other motifs to achieve NIR emission. ${ }^{48-52}$ The small size of the coumarin core would also likely require only minimal enzyme engineering to identify complementary luciferases. Given the unique structure of the CouLuc-1s, we further anticipated that the analogs could be used for multicomponent imaging with other red-emitting probes, including AkaLumine/Akaluc and furimazine/Antares.

Here we detail the synthesis and evaluation of the CouLuc-1 probes. We developed a two-step route to bridge the fluorescent coumarin heterocycle with the key thiazoline unit necessary for luciferin bioluminescence. The resulting conjugates displayed red-shifted emission. Complementary luciferases were identified via a parallel engineering approach. The resulting luciferase-luciferin pairs provided robust light outputs that were suitable for multiplexed imaging. Overall, these efforts provide a new class of easily accessible, long-wavelength bioluminescent pairs with significant promise for orthogonal imaging in vivo.

\section{RESULTS AND DISCUSSION}

Design and Synthesis of Coumarin-linked Luciferins. We set out to prepare CouLuc-1 analogs bearing different electron donors $\left(-\mathrm{NMe}_{2},-\mathrm{NH}_{2}\right.$, and $\left.\mathrm{OH}\right)$ at $\mathrm{C} 7$ of the coumarin heterocycle. These modifications are commonly found in coumarin fluorophores and are known to modulate the excited state properties. ${ }^{46}$ Retrosynthetic analysis of the CouLuc-1 analogs revealed the key disconnection in the alkene linkage between the coumarin and thiazoline (Figure 1c). We envisioned that if the olefin bridge could be installed with a nitrile handle, sub- sequent cysteine condensation would afford the desired luciferins in a highly concise two-step sequence. While methods to directly modify the carbonyl group on coumarins are rare, recent reports suggested that thiolactones are viable intermediates for synthesizing $\pi$-extended coumarins. ${ }^{48-49,53}$ Additionally, direct thiazoline formation from non-activated nitriles can be difficult, as similar transformations typically proceed through a multi-step sequence from corresponding carboxylates. $^{41,54}$

Scheme 1. Synthesis of coumarin luciferin scaffolds.

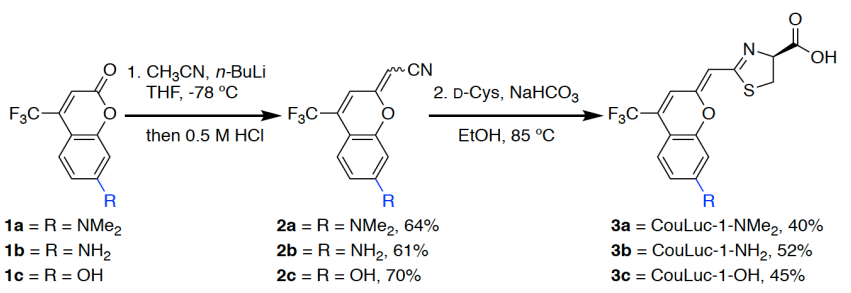

Reaction conditions: (1) 1 (1.0 equiv), $\mathrm{CH}_{3} \mathrm{CN}$ (4.0 equiv), $n$ BuLi (4.0 equiv), THF, $-78^{\circ} \mathrm{C}, 15 \mathrm{~min}$, then $0.5 \mathrm{M} \mathrm{HCl}, \mathrm{rt}, 1-4 \mathrm{~h}$; (2) 2 (1.0 equiv), D-Cys (1.5 equiv), $\mathrm{NaHCO}_{3}$ (4.0 equiv), $\mathrm{EtOH}$, $85^{\circ} \mathrm{C}, 80-120 \mathrm{~h}$

With these synthetic challenges in mind, we set out to develop olefination conditions for installing a cyanomethylene moiety onto commercial coumarin starting materials. After examining several strategies, we found that cyanomethyl anions generated in situ from acetonitrile and $n$-BuLi react readily with coumarins 1a-c. ${ }^{55}$ Subsequent treatment of the addition product with $0.5 \mathrm{M} \mathrm{HCl}$ afforded the desired cyanomethylene coumarins 2a-c as mixtures of $\mathrm{E} / \mathrm{Z}$ isomers in good yield (Scheme 1). Exposing 2a-c (as a mixture of isomers) to Dcysteine and $\mathrm{NaHCO}_{3}$ in ethanol generated CouLuc-1 analogs 3a-c in 3-5 days. Following cycloaddition, a single isomer was formed, and the geometry was verified by 2D NOSEY (Figure $\mathrm{S} 1$ ). Overall, the route provided access to 3a-c in $26-32 \%$ yield in just two steps. This approach is among the shortest luciferin syntheses to date from readily available starting materials. To highlight the scalability of the route, we also developed a chromatography-free procedure to access 3a (Figures S2-S3).

In Vitro Characterization with Native Luciferase. With the CouLuc-1 analogs in hand, we first examined their bioluminescent properties. All three analogs produced light when combined with Fluc and the necessary cofactors (Figure 2a). Scaffolds with amino (CouLuc-1- $\mathrm{NH}_{2}$,) or dimethylamino (CouLuc-1-NMe $\mathrm{N}_{2}$ ) substituents exhibited stronger photon outputs than the hydroxy variant (CouLuc-1-OH, Figure 2b). Similar boosts in brightness have been achieved with other amino luciferins. ${ }^{14,43}$ Compared to the native Fluc substrate (Dluc), though, the CouLuc-1 derivatives produced lower levels of light ( 1000-fold dimmer, Figure S4). The reduced brightness was partly attributed to low binding affinities between Fluc and the coumarin analogs (Figure S5). Similar trends have been observed with other sterically ${ }^{26,56}$ and electronically modified luciferins $\mathrm{s}^{34-35}$ exhibiting comparable emission levels (Figure S6). While low, the photon outputs achieved with Fluc and the CouLuc-1 derivatives provided a clear starting point for evolving brighter luciferases. 

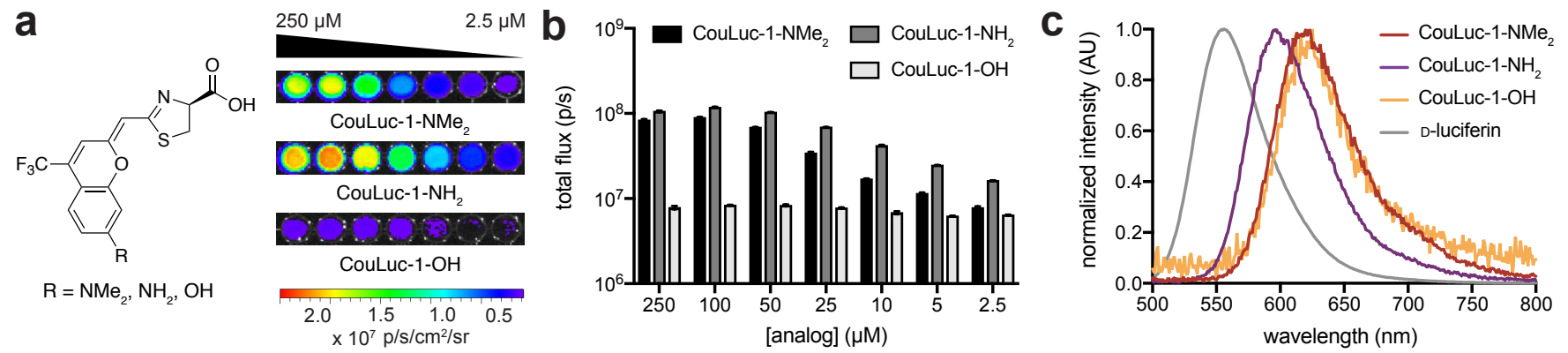

Figure 2. Light production from CouLuc-1 analogs. (a) Bioluminescence images from CouLuc-1 analogs $(2.5-250 \mu \mathrm{M})$ incubated with ATP $(100 \mu \mathrm{M})$, coenzyme A $(100 \mu \mathrm{M})$ and Fluc $(160 \mathrm{nM})$. (b) Quantification of the images from (a). Emission intensities are plotted as total photon flux values. Error bars represent the standard error of the mean for $n=3$ experiments. (c) Bioluminescence emission spectra for CouLuc-1 analogs.

In terms of color, CouLuc-1-NMe 2 and $-\mathrm{OH}$ exhibited peak emission wavelengths $\left(\lambda_{\max }\right)$ at 620 and $625 \mathrm{~nm}$, respectively. CouLuc-1- $\mathrm{NH}_{2}$ was slightly blue-shifted, with $\lambda_{\max }=597 \mathrm{~nm}$. Compared to D-luc, all analogs were red-shifted by $30-60 \mathrm{~nm}$ at $25{ }^{\circ} \mathrm{C}$ (Figure 2c). Nearly $40 \%$ of the emitted photons from CouLuc-1-NMe ${ }_{2}$ and CouLuc-1-OH were $>650 \mathrm{~nm}$. For comparison, Fluc/D-luc emits only $5 \%$ of photons $>650 \mathrm{~nm}$ in vitro $\left(8 \%\right.$ in cellulo $\left.{ }^{57}\right)$. For Akaluc/AkaLumine, a popular redshifted luciferase-luciferin pair, the value is $44 \%$ (in cellulo at $\left.25^{\circ} \mathrm{C}\right)$.

We further compared the CouLuc-1 bioluminescent spectra to the corresponding fluorescence spectra. In aqueous media, the fluorescence readouts exhibited similar trends, with the observed maxima red-shifted by $70-100 \mathrm{~nm}$ compared to D-luc (Table S1). This strong correlation between CouLuc-1 fluorescence and bioluminescence is consistent with other piextended and amino luciferins. ${ }^{28,39,58}$ CouLuc-1 fluorescence was also strongly influenced by solvent and $\mathrm{pH}$ (Figure S7, Table S2). In polar aprotic solvents, both amino CouLuc-1s were more blue-shifted (up to $170 \mathrm{~nm}$ ) than in protic or aqueous environments. Two peaks were observed in the emission spectrum for CouLuc-1-OH at acidic pH (517 and $600 \mathrm{~nm}$ ), likely due to different protonation states. Environmentally sensitive emission is common among coumarin derivatives. ${ }^{59}$ Such solvatochromatic behavior is attractive for bioluminescence imaging, as the "environment" can be tuned via engineering of the luciferase active site. ${ }^{60-61}$
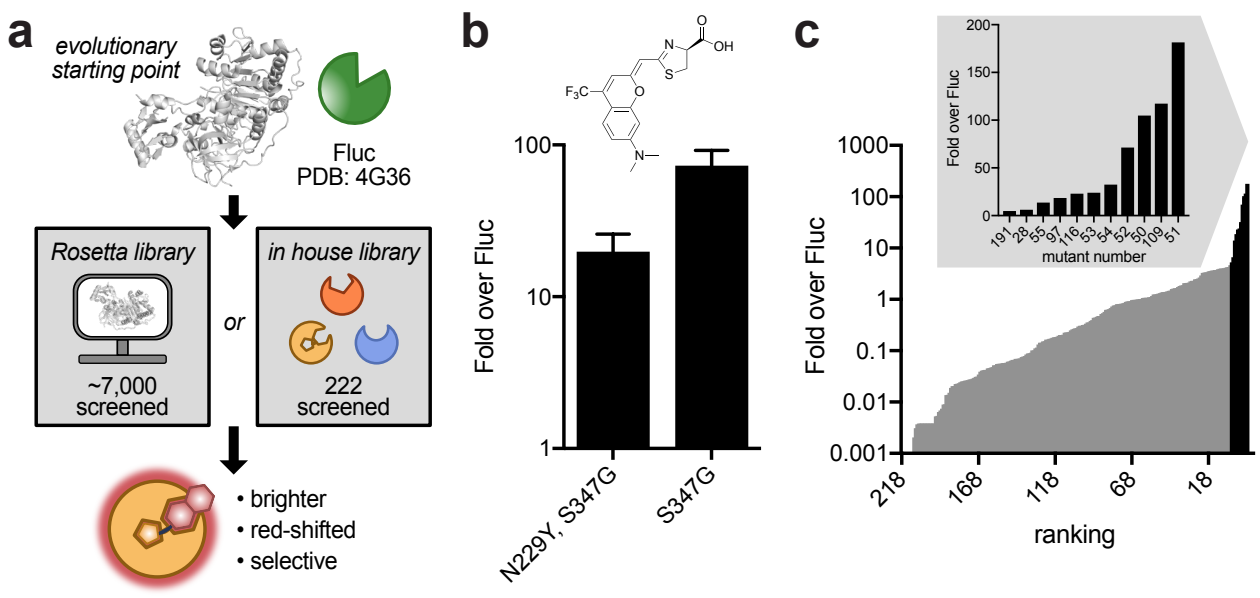

Engineering complementary luciferases for CouLuc-1s. After establishing the CouLuc-1s as viable luminophores, we set out to improve photon outputs by reengineering the luciferase enzyme (Figure 3a). The ideal mutants would exhibit improved turnover, maintain red-shifted emission, and be selective for the coumarin scaffolds. We initially focused on CouLuc-1-NMe $\mathrm{NMe}_{2}$ do its robust activity with Fluc and desirable spectral properties. We took a two-pronged screening approach to identifying mutants from library screens using (1) in silico design and (2) a semi-rational strategy. Both methods have been used to generate complementary enzymes for synthetic luciferins. ${ }^{26,39,45}$ In the first approach, we sculpted the luciferase active site using RosettaDesign. This strategy is useful for identifying mutations unique to an analog without significant prior knowledge or evolutionary starting point. ${ }^{62-63}$ To computationally identify mutations suited for the CouLuc1 scaffold, we used the Rosetta-Match algorithm ${ }^{64-65}$ to dock CouLuc-1-NMe ${ }_{2}$ with existing Fluc crystal structures. ${ }^{66}$ The model oriented the coumarin heterocycle toward the pocket normally adjacent to $\mathrm{C}^{\prime}$ ' on D-luc. In this configuration, the dimethylamino substituent was predicted to clash with the backbone of some active site residues (Figure S8), likely resulting in diminished turnover. We next employed RosettaDesign $^{64-65}$ to resolve this clash and optimize the packing interaction between the coumarin luciferin and surrounding residues. From this analysis, a total of 41 sites were mutated to create a complementary active site for CouLuc-1-NMe 2 (Fig-

Figure 3. Screening for complementary and bright luciferases. (a) A two-pronged engineering approach was pursued, featuring in silico and semi-rational library design. (b) Lead mutants identified from screening the Rosetta-inspired library. Luciferase expression was induced in bacteria and cultures were assayed with $100 \mu \mathrm{M}$ CouLuc-1-NMe $\mathrm{N}_{2}$ Relative light emission values are plotted as fold over the native enzyme (Fluc). Error bars represent the standard error of the mean for $n=3$ experiments. (c) Improved mutants identified from screening a focused library. Sequences of the top-ranked hits are listed. 
ure S9). These residues were then ranked for targeting based on their proximity and known biochemical data. ${ }^{27,67-69}$ Twenty sites were ultimately selected for randomization via combinatorial codon mutagenesis (Figure S9).

The resulting Rosetta-inspired library was introduced into bacteria, and the transformed colonies were sprayed with CouLuc-1-NMe $\mathrm{NM}_{2}$. Out of $\sim, 000$ colonies screened on plate, $\sim 150$ were light-emitting (Figure S10). These colonies were collected, and the mutants were verified in two secondary screens (Figure S10). Variants with $>10$-fold improved photon output over Fluc were carried forward. Unique sequences were then validated in a second bacterial cell assay. From this workflow, two hits were identified (Figure 3b). Intriguingly, both variants contained a $\mathrm{S} 347 \mathrm{G}$ mutation. We also screened the Rosetta-based library with CouLuc-1-OH. In this case, three hits were identified, with the point mutant S347G providing the most light (Figure S11). While only a subset of the Rosetta vo. ${ }^{29-30}$ When CouLuc-1-NMe $\mathrm{N}_{2}$ was incubated with purified Pecan, more intense light emission (77-fold) was observed compared to Fluc (Figure S13). The boost in light output was likely due to the enhanced binding affinity of CouLuc-1s with Pecan, as revealed by kinetic analyses (Figure $4 \mathrm{a}$ and $\mathrm{S} 14$ ). Importantly, robust emission in the NIR region was maintained. Approximately $30 \%$ of photons produced by CouLuc$1-\mathrm{NMe}_{2}$ were $>650 \mathrm{~nm}$, and the emission spectra for the other analogs were similarly red-shifted (Figure S15).

After examining Pecan/CouLuc-1-NMe 2 in vitro, we evaluated the pair in mammalian cells. Pecan and Fluc were transiently expressed in HEK293 cells. The cells were incubated with either the coumarin analogs or D-luc. Peak photon outputs for each enzyme-substrate combination were measured and normalized to a common transfection marker (GFP). As shown in Figures $4 \mathrm{~b}$ and S16, Pecan-expressing cells treated with CouLuc-1-NMe $\mathrm{NM}_{2}$ emitted 14-fold more photons than a

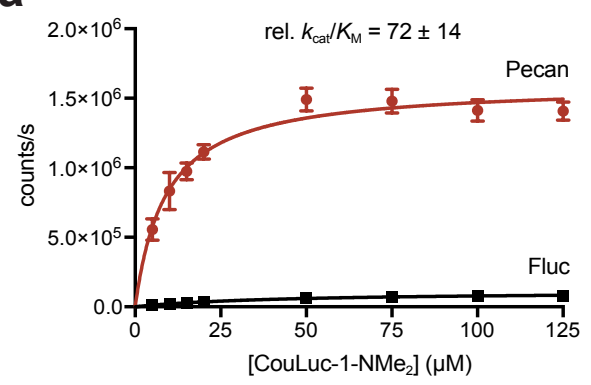

b

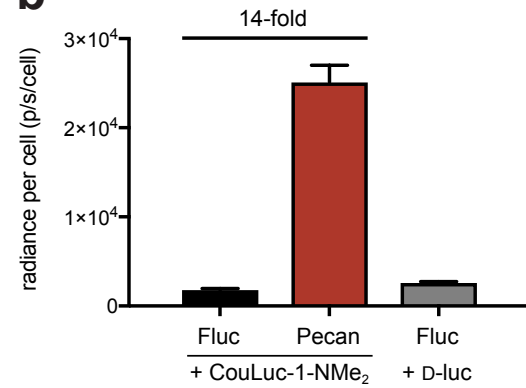

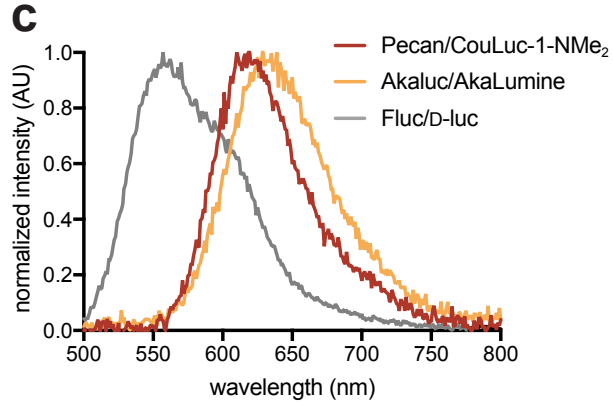

Figure 4. Photon output of CouLuc-1-NMe 2 with an engineered luciferase. (a) Kinetic studies revealed that Pecan could more efficiently process CouLuc-1-NMe 2 compared to Fluc. (b) Improved photon outputs were observed in cellulo. Pecan- or Fluc-expressing cells were incubated with either CouLuc-1-NMe $(250 \mu \mathrm{M})$ or D-luc $(250 \mu \mathrm{M})$. Transfection efficiencies were determined via co-expression of GFP. Peak emission intensities are plotted as photon flux per cell. Error bars represent the standard error of the mean for $n=3$ experiments. (c) In cellulo emission spectrum of Pecan/CouLuc-1-NMe 2 compared to other bioluminescent probes at $25^{\circ} \mathrm{C}$.

library was screened, the frequency of the S347G mutation among the 'hits' suggested that this residue is beneficial for CouLuc-1 processing. S347G is also known to stabilize the open conformation of the luciferase active site ${ }^{70-71}$ and process luciferins with steric bulk at $\mathrm{C}^{\prime}{ }^{26,29}$

In parallel with the Rosetta approach, we screened CouLuc$1-\mathrm{NMe}_{2}$ against a focused library of 222 previously characterized Fluc mutants. ${ }^{29}$ The luciferases comprise mutations confined to the luciferin binding pocket and exhibit unique preferences for sterically and electronically modified luciferins. ${ }^{26,39}$ Screens of this library could provide additional information on residues underlying substrate specificity for the CouLuc-1 scaffolds. All three analogs were subjected to the focused library. Eleven hits were identified for CouLuc-1-NMe 2 (Figure $3 \mathrm{c}$ ). A similar number of mutants were found to exhibit enhanced light emission for the $-\mathrm{NH}_{2}$ and $-\mathrm{OH}$ analogs (Figure $\mathrm{S} 12)$. The brightest mutants from these screens also comprised the S347G mutation, reinforcing the notion that residue 347 plays a pivotal role in CouLuc-1 processing. Another mutation common to multiple hits was F243M, a residue previously shown to aid in processing bulky luciferin analogs. ${ }^{29,56}$

In Vitro and In Cellulo Characterization of Lead Mutant. From the screening hits, we selected the F243M/S347G mutant (dubbed Pecan) for additional characterization with CouLuc-1-NMe 2 . Pecan was particularly attractive for orthogonal probe development as it has been previously used in vi-
Fluc-expressing cells. Similar improvements were observed when Pecan-expressing cells were incubated with either CouLuc-1-NH $\mathrm{NH}_{2}$ or CouLuc-1-OH (Figure S17). The robust emission from CouLuc-1 analogs was also recapitulated in Pecan-expressing DB7 cells (Figure S18). We next compared the emission from Pecan/CouLuc-1-NMe $\mathrm{N}_{2}$ to both native and engineered bioluminescent pairs (e.g. Fluc/CycLuc1 and Akaluc/AkaLumine). In both cases, the photon output compared favorably to the well-established bioluminescent pairs (Figure $\mathrm{S} 19)$. The Pecan/CouLuc-1-NMe 2 pair also provided significant numbers of NIR photons $(32 \%$ of photons $>650 \mathrm{~nm})$ in cells (Figure 4c). This level of NIR emission is on par with other state-of-the-art bioluminescent tools (Figure S20).

Multicellular imaging with Pecan and CouLuc-1-NMe The optical properties of the CouLuc-1 analogs coupled with their unique structures made them promising candidates for multiplexed imaging. As noted earlier, resolving luciferases by substrate requires unique luciferin architectures. The more structurally diverse the luciferins, the better they can be distinguished by engineered enzymes. ${ }^{29}$ Substrate unmixing and image processing algorithms can rapidly detect unique enzyme-substrate pairings within complex mixtures. ${ }^{30,72}$ Multiple classes of orthogonal probes are available for multicomponent imaging via these methods, but only a few exhibit the necessary optical properties (NIR bioluminescence) for sensitive imaging. 
Given the unique structures and robust emission of Pecan/CouLuc-1-NMe 2 , we reasoned that this pair would be useful for multiplexing with other red-shifted probes. We were drawn to Akaluc/AkaLumine ${ }^{45}$ and Antares/furimazine ${ }^{73}$ as these pairs have been recently used to monitor tumor-immune interactions in vivo. ${ }^{31}$ Pecan and Akaluc derive from the insect luciferase family and are thus inherently orthogonal to Antares, which uses a different mechanism for light emission. The CouLuc-1 scaffold is also structurally distinct from AkaLumine, suggesting Pecan and Akaluc could be readily differentiated based on their substrate preference. Indeed, minimal near infrared-emitting luciferins based on a modified coumarin scaffold. The substrates were synthesized in just 2-steps without chromatographic purification. The CouLuc-1 analogs were found to emit light with Fluc. While the emission levels were weak compared to existing bioluminescent systems, mutant luciferases were identified that afforded enhanced outputs. The brightest luciferase-CouLuc-1 pair exhibited luminescent signal on par with native bioluminescent probes. Such robust emission suggests that the CouLuc-1 probes can be immediately adopted for biological imaging. The unique structural and optical features of the CouLuc-1 analogs are further well

a

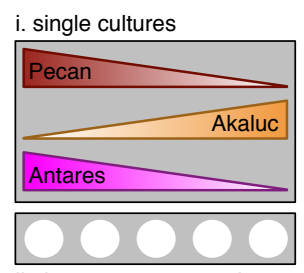

ii. three-component cultures

b

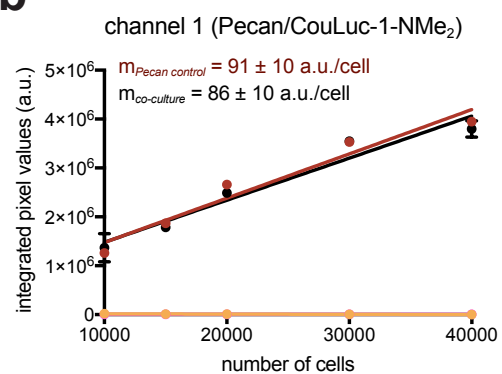

channel 1 (Pecan/ל̌r)
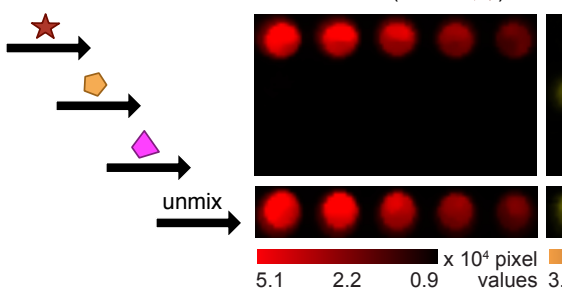

channel $2($ Akaluc/ $\square)$

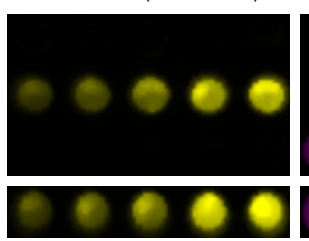

channel 3 (Antares/ $\triangle)$

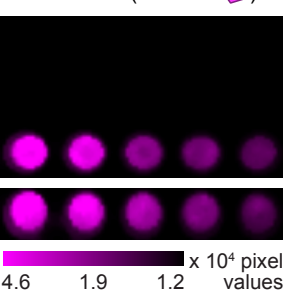

composite channel

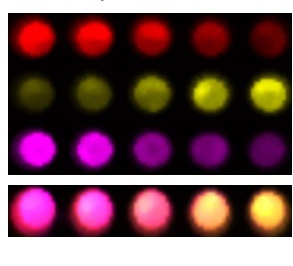

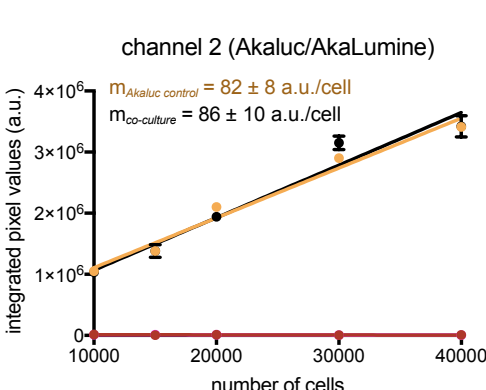

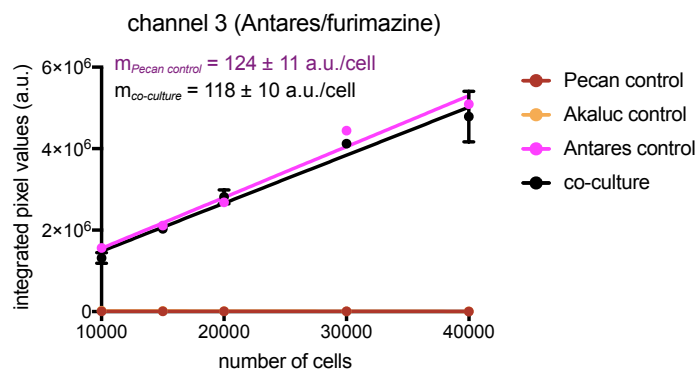

Figure 5. Multicomponent BLI with three NIR emitting probes. (a) Mixtures of Pecan-, Akaluc-, and Antares-expressing cells were plated in a 96-well pate. Luciferin analogs $(100 \mu \mathrm{M})$ were administrated sequentially with minimal delay time. BLI images were acquired, compiled, and processed via a linear unmixing algorithm. A final image was false colored to represent the proper luciferase-luciferin pairings. (b) Signal integrated from each colored pixel correlated with the number of cells plated. Values from each channel were fitted via linear regression. In channel $1, \mathrm{R}^{2}$ values for the Pecan control and co-culture wells are 0.95 and 0.95 , respectively. In channel $2, \mathrm{R}^{2}$ values for the Akaluc control and co-culture wells are 0.97 and 0.96, respectively. In channel 3, $\mathrm{R}^{2}$ values for the Antares control and co-culture wells are 0.98 and 0.98 , respectively. Error bars represent the standard error of the mean for $\mathrm{n}=3$ experiments.

crosstalk was observed when Pecan was treated with AkaLumine or when the CouLuc-1 luciferin was added to Akaluc (Figure S21). The high level of orthogonality highlights the unique chemical space occupied by the coumarin analogs.

To test the multiplexed strategy, we plated Pecan-, Akaluc-, and Antares-expressing DB7 cells in varying ratios. Cell cultures containing a single reporter were also plated as controls (Figure 5a). Each substrate was added sequentially, and an image was acquired after each administration. The resulting images were analyzed using a linear unmixing algorithm, ${ }^{30,74}$ and the relative abundance of each cell type was false colored in a composite image (Figure 5a). Each luciferase-expressing cell was readily discerned, and the unmixed signals correlated with the number of cells present (Figure 5b). The entire triple component imaging was completed within $30 \mathrm{~min}$, a notable improvement over traditional methods that require substrate clearance (multiple days). Altogether, these results suggest that Pecan/CouLuc-1-NMe $\mathrm{N}_{2}$ can be readily integrated with other engineered probes for rapid, multicellular imaging.

\section{Conclusion}

Despite the growing number of luciferin analogs, only a few are both chemically distinct and red-shifted. We have developed and characterized a new panel of structurally unique and suited for multiplexed imaging. Combinations of red-emitting bioluminescent probes are necessary for applications in tissue and other scattering environments.

More broadly, our approach to accessing novel luminophores from simple fluorophores could spur the development of an expanded set of bioluminescent tools. Future work will investigate whether additional architectures can be accessed using the olefination strategy. Additional luminophore tuning via restricted rotation and extended pi-conjugation will also be explored.

\section{ASSOCIATED CONTENT}

\section{Supporting Information}

The Supporting Information is available free of charge on the ACS Publications website.

Experimental details on luciferin synthesis, Rosetta analysis, library development, analog screens, and bioluminescence imaging are included.

\section{AUTHOR INFORMATION}




\section{Corresponding Author}

* (J.A.P) E-mail: jpresche@uci.edu; (M.J.S.) E-mail: martin.schnermann@nih.gov

\section{Author Contributions}

The manuscript was written through contributions of all authors. All authors have given approval to the final version of the manuscript. $\ddagger$ These authors contributed equally.

\section{Funding Sources}

The authors declare no competing financial interests.

\section{ACKNOWLEDGMENT}

This work was supported by the U.S. National Institutes of Health (R01 GM107630 to J.A.P.) and the Intramural Research Program of the National Institutes of Health (NIH), NCI-CCR. Z.Y. was supported by the National Science Foundation via the BEST IGERT (DGE-1144901) program and a Graduate Research Fellowship (DGE-1321846). A.L. was supported by the National Science Foundation via the BEST IGERT (DGE-1144901). We thank Dr. Joseph Barchi, NCI Center for Cancer Research (NCICCR) for NMR assistance and Dr. James Kelley, NCI-CCR, for mass spectrometry analysis. Some experiments were performed at the Laser Spectroscopy labs (LSL) at UCI. We thank Caroline Brennan for assisting with image processing, Anastasia Ionkina for cell line generation, along with other members of the Prescher lab for helpful discussions. We also thank members of the Weiss and Martin laboratories (UCI) for providing equipment and reagents.

\section{REFERENCES}

1. Paley, M. A.; Prescher, J. A. Bioluminescence: A versatile technique for imaging cellular and molecular features. MedChemComm 2014, 5, 255-267.

2. Mezzanotte, L.; van 't Root, M.; Karatas, H.; Goun, E. A.; Löwik, C. W. G. M. In Vivo Molecular Bioluminescence Imaging: New Tools and Applications. Trends Biotechnol. 2017, 35, 640-652.

3. Prescher, J. A.; Contag, C. H. Guided by the light: Visualizing biomolecular processes in living animals with bioluminescence. Curr. Opin. Chem. Biol. 2010, 14, 80-89.

4. Thorne, N.; Inglese, J.; Auld, D. S. Illuminating insights into firefly luciferase and other bioluminescent reporters used in chemical biology. Chem. Biol. 2010, 17, 646-657.

5. Ji, X.; Adams, S. T.; Miller, S. C., Chapter Eight Bioluminescence imaging in mice with synthetic luciferin analogues. In Methods Enzymol., Chenoweth, D. M., Ed. Academic Press: 2020; Vol. 640, pp 165-183.

6. Yeh, H.-W.; Ai, H.-W. Development and applications of bioluminescent and chemiluminescent reporters and biosensors. Annu. Rev. Anal. Chem. 2019, 12, 129-150.

7. Love, A. C.; Prescher, J. A. Seeing (and Using) the Light: Recent Developments in Bioluminescence Technology. Cell Chem. Biol. 2020, 27, 904-920.

8. Yao, Z.; Zhang, B. S.; Prescher, J. A. Advances in bioluminescence imaging: new probes from old recipes. Curr. Opin. Chem. Biol. 2018, $45,148-156$.

9. Rathbun, C. M.; Prescher, J. A. Bioluminescent probes for imaging biology beyond the culture dish. Biochemistry 2017, 56, 5178-5184.

10. Zambito, G.; Hall, M. P.; Wood, M. G.; Gaspar, N.; Ridwan, Y.; Stellari, F. F.; Shi, C.; Kirkland, T. A.; Encell, L. P.; Löwik, C.; Mezzanotte, L. Red-shifted click beetle luciferase mutant expands the multicolor bioluminescent palette for deep tissue imaging. iScience 2021, 24, 101986.

11. Markus, A.; Stefanie, V.; Cordula, S.; Amit, J.; Martin, P.; Mathias, H. Quantitative in vivo dual-color bioluminescence imaging in the mouse brain. Neurophotonics 2019, 6, 025006.
12. Mezzanotte, L.; Que, I.; Kaijzel, E.; Branchini, B.; Roda, A.; Löwik, C. Sensitive dual color in vivo bioluminescence imaging using a new red codon optimized firefly luciferase and a green click beetle luciferase. PLOS ONE 2011, 6, e19277.

13. Stowe, C. L.; Burley, T. A.; Allan, H.; Vinci, M.; Kramer-Marek, G.; Ciobota, D. M.; Parkinson, G. N.; Southworth, T. L.; Agliardi, G.; Hotblack, A.; Lythgoe, M. F.; Branchini, B. R.; Kalber, T. L.; Anderson, J. C.; Pule, M. A. Near-infrared dual bioluminescence imaging in mouse models of cancer using infraluciferin. eLife $\mathbf{2 0 1 9}, 8$, e45801.

14. Iwano, S.; Obata, R.; Miura, C.; Kiyama, M.; Hama, K.; Nakamura, M.; Amano, Y.; Kojima, S.; Hirano, T.; Maki, S.; Niwa, H. Development of simple firefly luciferin analogs emitting blue, green, red, and near-infrared biological window light. Tetrahedron 2013, 69, 3847-3856.

15. Yeh, H.-W.; Xiong, Y.; Wu, T.; Chen, M.; Ji, A.; Li, X.; Ai, H.W. ATP-Independent Bioluminescent Reporter Variants To Improve in Vivo Imaging. ACS Chem. Biol. 2019, 14, 959-965.

16. Sharma, D. K.; Adams, S. T.; Liebmann, K. L.; Choi, A.; Miller, S. C. Sulfonamides Are an Overlooked Class of Electron Donors in Luminogenic Luciferins and Fluorescent Dyes. Org. Lett. 2019, 21, $1641-1644$

17. Branchini, B. R.; Ablamsky, D. M.; Rosenman, J. M.; Uzasci, L.; Southworth, T. L.; Zimmer, M. Synergistic mutations produce blueshifted bioluminescence in firefly luciferase. Biochemistry 2007, 46, 13847-13855.

18. Branchini, B. R.; Ablamsky, D. M.; Murtiashaw, M. H.; Uzasci, L.; Fraga, H.; Southworth, T. L. Thermostable red and green lightproducing firefly luciferase mutants for bioluminescent reporter applications. Anal. Biochem. 2007, 361, 253-262.

19. Suzuki, K.; Kimura, T.; Shinoda, H.; Bai, G.; Daniels, M. J.; Arai, Y.; Nakano, M.; Nagai, T. Five colour variants of bright luminescent protein for real-time multicolour bioimaging. Nat. Commun. 2016, 7, 13718.

20. Takai, A.; Nakano, M.; Saito, K.; Haruno, R.; Watanabe, T. M.; Ohyanagi, T.; Jin, T.; Okada, Y.; Nagai, T. Expanded palette of Nanolanterns for real-time multicolor luminescence imaging. Proc. Natl. Acad. Sci. U. S. A. 2015, 112, 4352-4356.

21. Schaub, F. X.; Reza, M. S.; Flaveny, C. A.; Li, W.; Musicant, A. M.; Hoxha, S.; Guo, M.; Cleveland, J. L.; Amelio, A. L. FluorophoreNanoLuc BRET Reporters Enable Sensitive In Vivo Optical Imaging and Flow Cytometry for Monitoring Tumorigenesis. Cancer Research 2015, 75, 5023-5033.

22. Hiblot, J.; Yu, Q.; Sabbadini, M. D. B.; Reymond, L.; Xue, L.; Schena, A.; Sallin, O.; Hill, N.; Griss, R.; Johnsson, K. Luciferases with Tunable Emission Wavelengths. Angew. Chem. Int. Ed. 2017, $56,14556-14560$.

23. Rice, B. W.; Contag, C. H. The importance of being red. Nat. Biotechnol. 2009, 27, 624-625.

24. Weissleder, R.; Ntziachristos, V. Shedding light onto live molecular targets. Nat. Med. 2003, 9, 123-128.

25. Williams, S. J.; Prescher, J. A. Building biological flashlights: Orthogonal luciferases and luciferins for in vivo imaging. Acc. Chem. Res. 2019, 52, 3039-3050.

26. Jones, K. A.; Porterfield, W. B.; Rathbun, C. M.; McCutcheon, D. C.; Paley, M. A.; Prescher, J. A. Orthogonal luciferase-luciferin pairs for bioluminescence imaging. J. Am. Chem. Soc. 2017, 139, 23512358.

27. Adams, S. T.; Mofford, D. M.; Reddy, G. S. K. K.; Miller, S. C. Firefly luciferase mutants allow substrate-selective bioluminescence imaging in the Mouse Brain. Angew. Chem. Int. Ed. 2016, 55, 49434946.

28. Mofford, D. M.; Reddy, G. R.; Miller, S. C. Aminoluciferins extend firefly luciferase bioluminescence into the near-infrared and can be preferred substrates over D-luciferin. J. Am. Chem. Soc. 2014 , $136,13277-13282$.

29. Rathbun, C. M.; Porterfield, W. B.; Jones, K. A.; Sagoe, M. J.; Reyes, M. R.; Hua, C. T.; Prescher, J. A. Parallel screening for rapid identification of orthogonal bioluminescent tools. ACS Cent. Sci. 2017, 3, 1254-1261. 
30. Rathbun, C. M.; Ionkina, A. A.; Yao, Z.; Jones, K. A.; Porterfield, W. B.; Prescher, J. A. Rapid multicomponent bioluminescence imaging via substrate unmixing. ACS Chem. Biol. 2021, 16, 682-690.

31. Su, Y.; Walker, J. R.; Park, Y.; Smith, T. P.; Liu, L. X.; Hall, M. P.; Labanieh, L.; Hurst, R.; Wang, D. C.; Encell, L. P.; Kim, N.; Zhang, F.; Kay, M. A.; Casey, K. M.; Majzner, R. G.; Cochran, J. R.; Mackall, C. L.; Kirkland, T. A.; Lin, M. Z. Novel NanoLuc substrates enable bright two-population bioluminescence imaging in animals. Nat. Methods 2020, 17, 852-860.

32. Hall, M. P.; Woodroofe, C. C.; Wood, M. G.; Que, I.; van't Root, M.; Ridwan, Y.; Shi, C.; Kirkland, T. A.; Encell, L. P.; Wood, K. V.; Löwik, C.; Mezzanotte, L. Click beetle luciferase mutant and near infrared naphthyl-luciferins for improved bioluminescence imaging. Nat. Commun. 2018, 9, 132.

33. Anderson, J. C.; Grounds, H.; Jathoul, A. P.; Murray, J. A. H.; Pacman, S. J.; Tisi, L. Convergent synthesis and optical properties of near-infrared emitting bioluminescent infra-luciferins. RSC Adv. 2017, 7, 3975-3982.

34. McCutcheon, D. C.; Paley, M. A.; Steinhardt, R. C.; Prescher, J. A. Expedient synthesis of electronically modified luciferins for bioluminescence imaging. J. Am. Chem. Soc. 2012, 134, 7604-7607.

35. Zhang, B. S.; Jones, K. A.; McCutcheon, D. C.; Prescher, J. A. Pyridone luciferins and mutant luciferases for bioluminescence imaging. ChemBioChem 2018, 19, 470-477.

36. Woodroofe, C. C.; Meisenheimer, P. L.; Klaubert, D. H.; Kovic, Y.; Rosenberg, J. C.; Behney, C. E.; Southworth, T. L.; Branchini, B. R. Novel Heterocyclic Analogues of Firefly Luciferin. Biochemistry 2012, 51, 9807-9813.

37. Branchini, B. R., Chemical synthesis of firefly luciferin analogs and inhibitors. In Methods Enzymol., Academic Press: 2000; Vol. 305, pp 188-195.

38. Takakura, H.; Sasakura, K.; Ueno, T.; Urano, Y.; Terai, T.; Hanaoka, K.; Tsuboi, T.; Nagano, T. Development of Luciferin Analogues Bearing an Amino Group and Their Application as BRET Donors. Chem. Asian. J. 2010, 5, 2053-2061.

39. Yao, Z.; Zhang, B. S.; Steinhardt, R. C.; Mills, J. H.; Prescher, J. A. Multicomponent Bioluminescence Imaging with a $\pi$-Extended Luciferin. J. Am. Chem. Soc. 2020, 142, 14080-14089.

40. Kitada, N.; Saitoh, T.; Ikeda, Y.; Iwano, S.; Obata, R.; Niwa, H.; Hirano, T.; Miyawaki, A.; Suzuki, K.; Nishiyama, S.; Maki, S. A. Toward bioluminescence in the near-infrared region: Tuning the emission wavelength of firefly luciferin analogues by allyl substitution. Tetrahedron Lett. 2018, 59, 1087-1090.

41. Jathoul, A. P.; Grounds, H.; Anderson, J. C.; Pule, M. A. A dualcolor far-red to near-infrared firefly luciferin analogue designed for multiparametric bioluminescence imaging. Angew. Chem. Int. Ed. 2014, 53, 13059-13063.

42. Ikeda, Y.; Nomoto, T.; Hiruta, Y.; Nishiyama, N.; Citterio, D Ring-Fused Firefly Luciferins: Expanded Palette of Near-Infrared Emitting Bioluminescent Substrates. Anal. Chem. 2020, 92, 42354243.

43. Reddy, G. R.; Thompson, W. C.; Miller, S. C. Robust Light Emission from Cyclic Alkylaminoluciferin Substrates for Firefly Luciferase. J. Am. Chem. Soc. 2010, 132, 13586-13587.

44. Anderson, J. C.; Chang, C.-H.; Jathoul, A. P.; Syed, A. J. Synthesis and bioluminescence of electronically modified and rotationally restricted colour-shifting infraluciferin analogues. Tetrahedron 2019, 75, 347-356.

45. Iwano, S.; Sugiyama, M.; Hama, H.; Watakabe, A.; Hasegawa, N.; Kuchimaru, T.; Tanaka, K. Z.; Takahashi, M.; Ishida, Y.; Hata, J.; Shimozono, S.; Namiki, K.; Fukano, T.; Kiyama, M.; Okano, H.; Kizaka-Kondoh, S.; McHugh, T. J.; Yamamori, T.; Hioki, H.; Maki, S.; Miyawaki, A. Single-cell bioluminescence imaging of deep tissue in freely moving animals. Science 2018, 359, 935-939.

46. Cao, D.; Liu, Z.; Verwilst, P.; Koo, S.; Jangjili, P.; Kim, J. S.; Lin, W. Coumarin-Based Small-Molecule Fluorescent Chemosensors. Chem. Rev. 2019, 119, 10403-10519.

47. Sednev, M. V.; Belov, V. N.; Hell, S. W. Fluorescent dyes with large Stokes shifts for super-resolution optical microscopy of biological objects: a review. Methods and Applications in Fluorescence 2015, 3, 042004.

48. Gandioso, A.; Contreras, S.; Melnyk, I.; Oliva, J.; Nonell, S.; Velasco, D.; García-Amorós, J.; Marchán, V. Development of Green/Red-Absorbing Chromophores Based on a Coumarin Scaffold That Are Useful as Caging Groups. J. Org. Chem. 2017, 82, 53985408.

49. Gandioso, A.; Bresolí-Obach, R.; Nin-Hill, A.; Bosch, M.; Palau, M.; Galindo, A.; Contreras, S.; Rovira, A.; Rovira, C.; Nonell, S.; Marchán, V. Redesigning the Coumarin Scaffold into Small Bright Fluorophores with Far-Red to Near-Infrared Emission and Large Stokes Shifts Useful for Cell Imaging. J. Org. Chem. 2018, 83, 11851195 .

50. Matikonda, S. S.; Ivanic, J.; Gomez, M.; Hammersley, G.; Schnermann, M. J. Core remodeling leads to long wavelength fluorocoumarins. Chem. Sci. 2020, 11, 7302-7307.

51. Lei, Z.; Sun, C.; Pei, P.; Wang, S.; Li, D.; Zhang, X.; Zhang, F. Stable, Wavelength-Tunable Fluorescent Dyes in the NIR-II Region for In Vivo High-Contrast Bioimaging and Multiplexed Biosensing. Angew. Chem. Int. Ed. 2019, 58, 8166-8171.

52. Chen, H.; Dong, B.; Tang, Y.; Lin, W. A Unique "Integration" Strategy for the Rational Design of Optically Tunable Near-Infrared Fluorophores. Acc. Chem. Res. 2017, 50, 1410-1422.

53. Abdel-Fattah Mostafa, A.; SathishKumar, C.; Al-Askar, A. A.; Sayed, S. R. M.; SurendraKumar, R.; Idhayadhulla, A. Synthesis of novel benzopyran-connected pyrimidine and pyrazole derivatives via a green method using $\mathrm{Cu}$ (ii)-tyrosinase enzyme catalyst as potential larvicidal, antifeedant activities. RSC Adv . 2019, 9, 25533-25543.

54. Kuchimaru, T.; Iwano, S.; Kiyama, M.; Mitsumata, S.; Kadonosono, T.; Niwa, H.; Maki, S.; Kizaka-Kondoh, S. A luciferin analogue generating near-infrared bioluminescence achieves highly sensitive deep-tissue imaging. Nat. Commun. 2016, 7, 11856.

55. Bianchi, G.; Feroci, M.; Rossi, L. Reaction of the Electrogenerated Cyanomethyl Anion with Carbonyl Compounds: A Clean and Safe Synthesis of $\beta$-Hydroxynitriles. Eur. J. Org. Chem. 2009, 2009, 3863-3866.

56. Williams, S. J.; Hwang, C. S.; Prescher, J. A. Orthogonal Bioluminescent Probes from Disubstituted Luciferins. Biochemistry 2021, 60, 563-572.

57. Zhao, H.; Doyle, T. C.; Coquoz, O.; Kalish, F.; Rice, B. W.; Contag, C. H. Emission spectra of bioluminescent reporters and interaction with mammalian tissue determine the sensitivity of detection in vivo. J. Biomed. Opt. 2005, 10, 41210.

58. Vreven, T.; Miller, S. C. Computational investigation into the fluorescence of luciferin analogues. J. Comput. Chem. 2019, 40, 527531.

59. Signore, G.; Nifosì, R.; Albertazzi, L.; Storti, B.; Bizzarri, R. Polarity-Sensitive Coumarins Tailored to Live Cell Imaging. $J$. Am. Chem. Soc. 2010, 132, 1276-1288.

60. Morton, R. A.; Hopkins, T. A.; Seliger, H. H. Spectroscopic properties of firefly luciferin and related compounds; an approach to product emission. Biochemistry 1969, 8, 1598-1607.

61. Bevilaqua, V. R.; Matsuhashi, T.; Oliveira, G.; Oliveira, P. S. L.; Hirano, T.; Viviani, V. R. Phrixotrix luciferase and 6'-aminoluciferins reveal a larger luciferin phenolate binding site and provide novel farred combinations for bioimaging purposes. Sci. Rep. 2019, 9, 8998.

62. Liu, D. S.; Nivón, L. G.; Richter, F.; Goldman, P. J.; Deerinck, T. J.; Yao, J. Z.; Richardson, D.; Phipps, W. S.; Ye, A. Z.; Ellisman, M. H.; Drennan, C. L.; Baker, D.; Ting, A. Y. Computational design of a red fluorophore ligase for site-specific protein labeling in living cells. Proc. Natl. Acad. Sci. U.S. A. 2014, 111, E4551-E4559.

63. Pearson, A. D.; Mills, J. H.; Song, Y.; Nasertorabi, F.; Han, G. W.; Baker, D.; Stevens, R. C.; Schultz, P. G. Trapping a transition state in a computationally designed protein bottle. Science $\mathbf{2 0 1 5}, 347$, $863-867$.

64. Tinberg, C. E.; Khare, S. D.; Dou, J.; Doyle, L.; Nelson, J. W.; Schena, A.; Jankowski, W.; Kalodimos, C. G.; Johnsson, K.; Stoddard, B. L.; Baker, D. Computational design of ligand-binding proteins with high affinity and selectivity. Nature 2013, 501, 212216. 
65. Zanghellini, A.; Jiang, L.; Wollacott, A. M.; Cheng, G.; Meiler, J.; Althoff, E. A.; Röthlisberger, D.; Baker, D. New algorithms and an in silico benchmark for computational enzyme design. Protein Sci. 2006, $15,2785-2794$.

66. Sundlov, J. A.; Fontaine, D. M.; Southworth, T. L.; Branchini, B. R.; Gulick, A. M. Crystal Structure of Firefly Luciferase in a Second Catalytic Conformation Supports a Domain Alternation Mechanism. Biochemistry 2012, 51, 6493-6495.

67. Viviani, V. R.; Simões, A.; Bevilaqua, V. R.; Gabriel, G. V. M.; Arnoldi, F. G. C.; Hirano, T. Glu311 and Arg337 stabilize a closed active-site conformation and provide a critical catalytic base and countercation for green bioluminescence in beetle Luciferases. Biochemistry 2016, 55, 4764-4776.

68. Branchini, B. R.; Magyar, R. A.; Murtiashaw, M. H.; Anderson, S. M.; Helgerson, L. C.; Zimmer, M. Site-Directed Mutagenesis of Firefly Luciferase Active Site Amino Acids: A Proposed Model for Bioluminescence Color. Biochemistry 1999, 38, 13223-13230.

69. Choudhury, A. K.; Golovine, S. Y.; Dedkova, L. M.; Hecht, S. M. Synthesis of Proteins Containing Modified Arginine Residues. Biochemistry 2007, 46, 4066-4076.

70. Branchini, B. R.; Southworth, T. L.; Murtiashaw, M. H.; Boije, H.; Fleet, S. E. A mutagenesis study of the putative luciferin binding site residues of firefly luciferase. Biochemistry 2003, 42, 1042910436.

71. Harwood, K. R.; Mofford, D. M.; Reddy, G. R.; Miller, S. C. Identification of mutant firefly luciferases that efficiently utilize aminoluciferins. Chem. Biol. 2011, 18, 1649-1657.

72. Sarrion-Perdigones, A.; Chang, L.; Gonzalez, Y.; Gallego-Flores, T.; Young, D. W.; Venken, K. J. T. Examining multiple cellular pathways at once using multiplex hextuple luciferase assaying. Nat. Commun. 2019, 10, 5710.

73. Chu, J.; Oh, Y.; Sens, A.; Ataie, N.; Dana, H.; Macklin, J. J.; Laviv, T.; Welf, E. S.; Dean, K. M.; Zhang, F.; Kim, B. B.; Tang, C. T.; Hu, M.; Baird, M. A.; Davidson, M. W.; Kay, M. A.; Fiolka, R.; Yasuda, R.; Kim, D. S.; Ng, H.-L.; Lin, M. Z. A bright cyan-excitable orange fluorescent protein facilitates dual-emission microscopy and enhances bioluminescence imaging in vivo. Nat. Biotechnol. 2016, 34, 760-767.

74. Gammon, S. T.; Leevy, W. M.; Gross, S.; Gokel, G. W.; PiwnicaWorms, D. Spectral unmixing of multicolored bioluminescence emitted from heterogeneous biological sources. Anal. Chem. 2006, 78, 1520-1527.

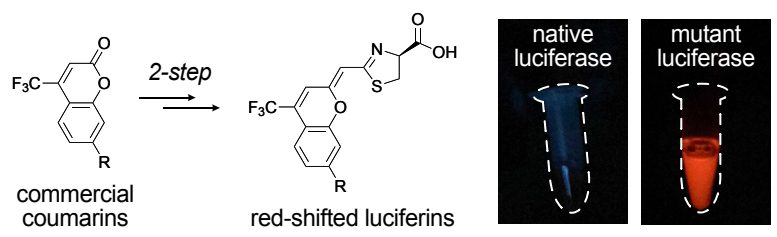

Table of Contents artwork 